\title{
Caractériser l'attractivité d'un territoire, une approche par les ressources : Le cas de 5 EMN européennes nouvellement implantées dans la région de Kalouga en Russie.
}

Cet article s'intéresse à l'attractivité d'un territoire : la région de Kalouga en Russie. L'objectif porte sur le lien théorique et empi-rique entre action publique locale et localisa-tion des entreprises multinationales (EMN) pour en expliquer l'attractivité. L'approche choisie est celle des ressources échangées afin de mettre en perspective l'action publique en matière d'attractivité (Colletis et Pecqueur, 2005). L'étude empirique est fondée sur l'ana-lyse de 5 processus d'implantation d'EMN européennes dans la région de Kalouga. Les résultats révèlent que les ressources territo-riales décisives mises à disposition des EMN par les acteurs territoriaux sont de nature idéelle, informationnelle et relationnelle. La qualité des pratiques de management terri-torial devient donc un facteur d'attractivité territoriale non négligeable.

Mots clés : Management Public et attractivité territoriale, EMN, acteurs territoriaux, ressources territoriales.

\section{Characterize the Attractiveness of a Territory, a Resources-base Approach: The Case of Five European MNEs Newly Located in the Kaluga Region of Russia.}

This article focuses on the theoretical and empirical link between local public action and the locational process of Multi-National Companies (MNC). Through a qualitative study, based on five MNC recently estab-lished in Kalouga (Russian region), the results underline the nature and the role of local public resources : among location factors, territorial management practices become more and more important in a con-text of high uncertainty.

Keywords: Public management and territorial attractiveness, MNE, territorial actors, territorial resources.

\section{Caracterizar el atractivo de un territorio, un enfoque de recursos : El caso de cinco empresas multinacionales europeas recientemente localizadas en la región de Kaluga, Rusia.}

\begin{abstract}
Este artículo trata de la capacidad de atracción de un territorio : la región de Kaluga de Rusia. El objetivo se centra en la relación teórica y empírica entre la acción pública local y la localización de empresas multinacionales (EMN ) para explicar la atracción. El enfoque elegido es el de los recursos intercambiados para poner en perspectiva ela relación entre acción pública y atractivo territorial ( Colletis y Pecqueur, 2005). El estudio empírico se basa en el análisis del proceso de implementación de 5 empresas multinacionales europeas en la región de Kaluga. Los resultados revelan que los recursos territoriales decisivos faci-litados a las empresas multinacionales son actores territoriales de tipo ideal, informativo y relacional. La calidad de las prácticas de gestión del territorio se convierte en un factor atractivo territorial considerable.
\end{abstract}

Palabras clave : Gestión Pública y el atrac-

tivo territorial, la REM, los actores locales,

los recursos locales.

$\begin{array}{lll}\text { OLIVIER KERAMIDAS } & \text { EKATERINA LE PENNEC } & \text { SARAH SERVAL } \\ \text { Aix Marseille Université, } & \text { Université Savoie Mont Blanc, IREGE, } & \text { Université Paris-Sud, Université Paris- } \\ \text { IMPGT-CERGAM, EA4225 } & \text { EA } 2426 & \text { Saclay, RITM, EA7360 }\end{array}$

T 'attractivité des territoires est devenue un objectif des straLtégies territoriales mises en œuvre par des acteurs publics et privés (Noisette et Valérugo, 2010). L'environnement concurrentiel est prégnant et l'attractivité s'impose comme un indicateur de performance du territoire (Keramidas 2012; Hatem, 2005). Cet article s'intéresse à l'attractivité d'un territoire «chemin faisant», à travers l'étude du processus d'implantation d'Entre- prises Multi-Nationales (EMN) et le rôle joué par les acteurs territoriaux. L'objet de cette recherche porte donc sur le lien théorique et empirique entre action publique locale et localisation des EMN pour expliquer l'attractivité d'un territoire.

L'espace territorial demeure un facteur peu présent dans les travaux en management public et en management international (malgré le célèbre article de Dunning en 1998). Il est 
étudié à un niveau national en opposant généralement le pays d'origine et le pays d'accueil (Beugelsdijk et Mudambi, 2013; Kim et Aguilera, 2015). Cette focalisation s'explique par le rôle historique des frontières nationales, l'importance des gouvernements nationaux dans le commerce mondial, le poids des environnements institutionnels nationaux dans la formulation des stratégies des EMN mais aussi par la disponibilité des données à ce niveau d'analyse (Beugelsdijk et Mudambi, 2013). Pour autant, sous l'influence des travaux issus de la Nouvelle Economie Géographique, les études suggèrent que les échelons territoriaux infranationaux ont un impact sur les comportements de localisation (McCann et Mudambi, 2005; Rugman et Verbeke, 2009; Narula et Dunning, 2010; Meyer et al., 2011; Rugman et al., 2011). Dans cette perspective, les recherches récentes mettent en avant les nuances infranationales, particulièrement au sein des économies émergeantes comme les BRICS. Dans ce contexte spécifique, les territoires infranationaux d'une part sont très hétérogènes, et drautre part disposent de gouvernements locaux ayant un pouvoir considérable dans les politiques d'attractivité d'Investissements Directs Etrangers comme la Russie qui fédère plus de 80 unités autonomes (Chan et al., 2010; Ma et al., 2010; Ma et al., 2013).

$\mathrm{Au}$ sein des pays émergeants, le territoire infranational devient un facteur explicatif à la fois des décisions de localisation (Meyer et Nguyen, 2005; Mataloni, 2011; Beugelsdijk et Mudambi, 2013) et du différentiel de performance entre les filiales étrangères des EMN (Chan et al., 2010; Ma et al., 2013). Parmi les caractéristiques de l'environnement local, la littérature a identifié l'action publique locale comme un déterminant des décisions de localisation (Sachwald et Perrin, 2003; Sergot, 2004; Le Gall, 2009) et plus généralement comme une caractéristique de l'environnement local qui peut faciliter l'action d'une EMN sur un territoire (Andersson et al., 2002; Verbeke et al., 2007; Rugman et Verbeke, 2009; Rugman et al., 2011). Les relations entre EMN et acteurs publics locaux influencent positivement le comportement de localisation des EMN à un niveau infranational (Meyer et Nguyen, 2005; Meyer et al., 2011). Certains gouvernements locaux cherchent à se différencier et mènent des réformes locales visant à développer des dispositifs spécifiques pour attirer des EMN étrangères comme en Russie ou en Inde (Meyer et Nguyen, 2005). Néanmoins, la littérature en management public reste discrète sur la manière dont cette action publique locale prend forme, sur les éléments de cette action publique qui expliquent en partie le succès des processus d'implantation des EMN étrangères sur un territoire (Coussi, 2014), et particulièrement dans le contexte spécifique des pays émergeants (Meyer et Nguyen, 2005).

L'attractivité territoriale est entendue «comme la capacité d'un territoire à être choisi par un acteur comme zone de localisation (temporaire ou durable) pour tout ou partie de ses activités » (Gérardin et Poirot, 2010 : 27). Il s'agit d'étudier la rencontre productive entre territoire et EMN, à travers l'étude des interactions entre acteurs territoriaux et EMN, lors du processus d'implantation, par le prisme des ressources échangées afin de mettre en perspective l'action publique en matière d'attractivité (Colletis et Pecqueur, 2005). En interprétant le niveau de territorialité de la ressource engagée, et donc son caractère plus ou moins spécifique, il est possible de comprendre quels sont les dispositifs publics et les processus managériaux mis en œuvre par les acteurs territoriaux, les plus à mêmes d'expliquer la réussite des processus d'implantation d'EMN et la construction d'une attractivité territoriale spécifique. Ainsi, nous cherchons à répondre à la question suivante : En quoi l'action publique locale favorise-t-elle l'attraction d'EMN étrangères? Nous définissons dans un premier temps le concept d'attractivité territoriale ainsi que les modalités d'interventions publiques afférentes. Nous développons également l'approche adoptée pour comprendre les interactions entre acteurs territoriaux et EMN, et leur niveau de territorialité qui explique et caractérise l'attractivité du territoire étudié (Colletis et Pecqueur, 1993).

De manière connexe à nos recherches en Management Public, les travaux publiés en Management International permettent d'appréhender l'intégration de la dimension locale qui se fait généralement à travers une perspective institutionnaliste ou une approche en termes de ressources (Meyer et al., 2011). Cet article s'insère dans la deuxième approche et mobilise un corpus théorique davantage marginal en management international, fondé sur les travaux des géographes économiques, et plus précisément sur l'école de la proximité. Les recommandations de Beugelsdijk et Mudambi (2013) visent à mobiliser ce corpus théorique afin de compléter les travaux portant sur les décisions de localisation des EMN à travers l'angle de vue du territoire.

Nous présentons ensuite une méthodologie reposant sur une étude de cas unique enchâssée menée sur la région de Kalouga en Russie, où nous avons étudié les processus d'implantation de cinq EMN européennes. Enfin, nous présentons et discutons les résultats qui révèlent une forte implication des acteurs publics locaux dans les différents processus d'implantation. Les ressources mises à disposition des EMN par les acteurs territoriaux, et qui apparaissent décisives dans la localisation des EMN, sont de natures idéelle, informationnelle et relationnelle. In fine, les ressources financières et la question du coût lié à la localisation ne sont pas présentées comme des ressources critiques par les acteurs interrogés pour expliquer leur choix final de localisation et la réussite de leur implantation. En revanche, l'effort consenti par les acteurs publics locaux pour construire une relation avec les EMN, leur implication, leur expérience en matière d'accompagnement dans le processus d'implantation, leurs compétences en termes de coordination et de gouvernance territoriale, apparaissent comme des ressources spécifiques qui ont fait la différence et contribuent à construire l'attractivité et la réputation du territoire de Kalouga. Les acteurs publics locaux sont présentés par les EMN comme de puissants réducteurs d'incertitude. En ce sens, la qualité des pratiques de management territorial devient un facteur explicatif de la réussite du processus d'implantation d'une EMN, et partant un facteur d'attractivité territoriale non négligeable.

\section{L’Attractivité des territoires et l'implantation d'EMN : une logique publique de ressources territoriales}

\section{LE CONCEPT D'ATTRACTIVITÉ TERRITORIALE AU CGUR DE L'ANALYSE}

La notion d'attractivité territoriale résulte de l'importance des entreprises multinationales dans l'économie et de la dépendance 
FIGURE 1

Les trois composantes qui influencent les perceptions d'une EMN vis-à-vis de l'attractivité d'un territoire

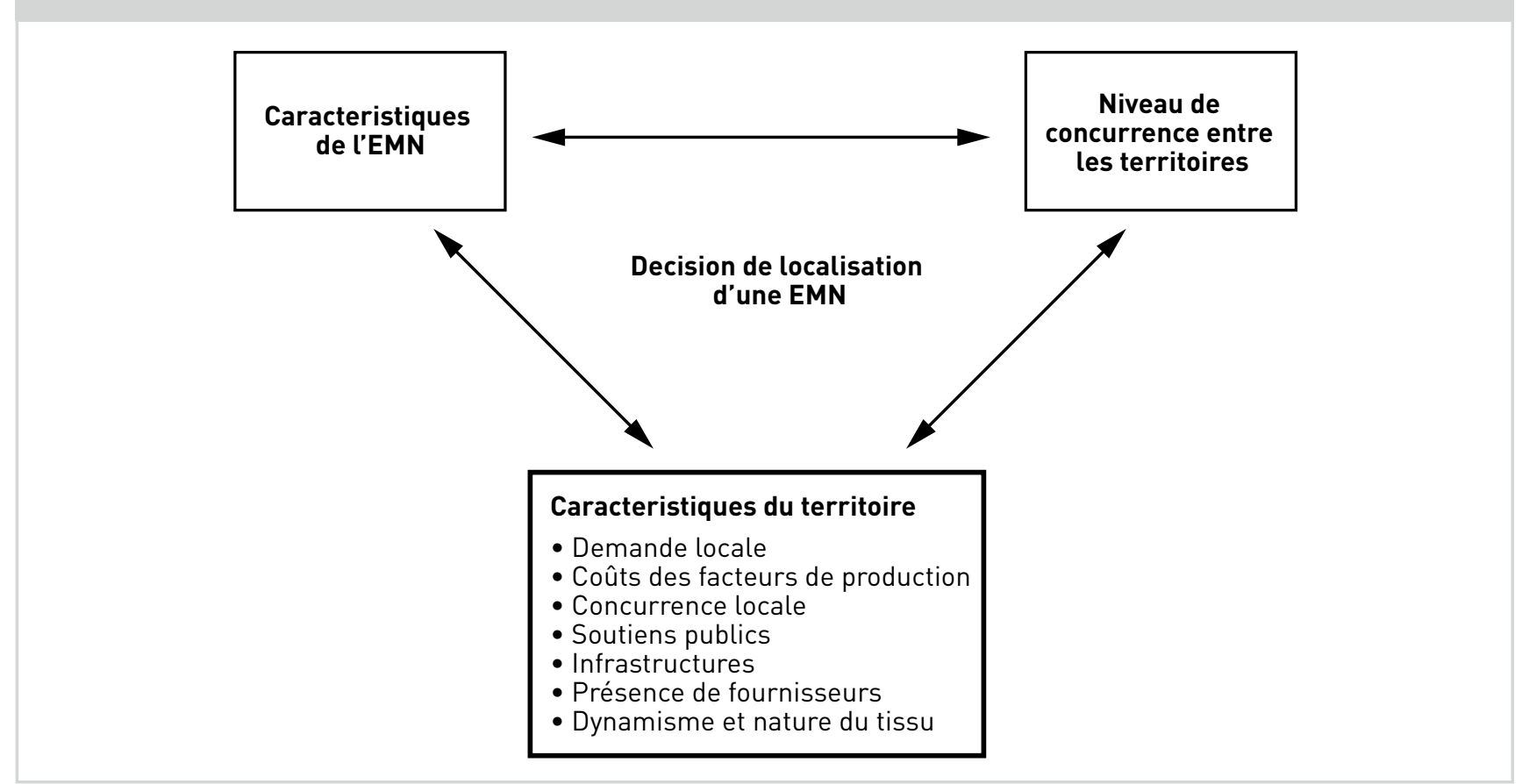

Source : Auteurs

des territoires par rapport à leur choix de localisation (Hatem, 2005). L'entreprise est qualifiée de multinationale (EMN) dans la mesure où elle opère une localisation à l'international, dans au moins deux pays, de tout ou partie de sa chaîne de valeur (Krugman, 1995). Cette localisation est définie comme «le choix des firmes de faire faire hors des frontières nationales ce qu'elles auraient pu faire dans leur pays d'origine (Mucchielli, 1998)» (Colovic et Mayrhofer, 2008 : 153). Loin d'être figée, l'attractivité territoriale est un concept multidimensionnel et dynamique qui se définit «comme la capacité d`un territoire à être choisi par un acteur comme zone de localisation (temporaire ou durable) pour tout ou partie de ses activités » (Gérardin et Poirot, 27 : 2010).

Témoignant d'une montée du pragmatisme économique, l'importance accordée à l'attractivité des EMN repose sur le lien théorique et empirique entre EMN et croissance économique d'un pays (Sachwald et Perrin, 2003). Les politiques publiques d'attractivité territoriale visent depuis les années 1980 à attirer les investisseurs étrangers. Cette orientation résulte d'un glissement paradigmatique des avantages compétitifs des entreprises aux avantages compétitifs des territoires, désormais inscrits dans un environnement concurrentiel (Porter, 1990). Cette concurrence territoriale amène la création "d'une offre de site» (Jayet, 1993) et l'émergence d'un "marché de l'implantation» (Hatem, 2005). Ainsi, l'internationalisation des entreprises n'est plus une crainte en soi; elle est intégrée dans les préoccupations des acteurs publics qui orientent leurs stratégies vers l'attraction des EMN (Ferrara et Henriot, 2004; Carluer, 2005). On passe ici d'une logique interventionniste en faveur de la compétitivité des entreprises à une logique interventionniste orientée vers l'augmentation de l'attractivité des territoires (Crozet et Mayer, 2002). Les acteurs publics sont donc en charge de construire les conditions nécessaires pour le développement économique local et l'implantation d'entreprises (Porter, 2000).

L'attractivité d'un territoire sera évaluée par une entreprise au regard de trois composantes (figure 1 p.25) : «les caractéristiques de la demande [de l'entreprise] (...), les caractéristiques du territoire lui-même et l'intensité de la concurrence entre les territoires» (Gérardin et Poirot, 32 : 2010). En ce sens, les «avantages de première nature» ne suffisent pas à expliquer l'attractivité du territoire (Krugman, 1993). Les facteurs d`attractivité, pour un investisseur, sont donc multiples et contextuels, correspondant à une dialectique complexe entre les dimensions de l>offre recherchée, son coût et le niveau de qualité souhaité. Les dimensions de l`offre sont diverses (main d'œuvre, infrastructures du territoire, services disponibles, fournisseurs, existence d'un marché et d'une demande potentielle, coût des facteurs de production, dynamisme et nature du tissu économique local, ainsi que les orientations des dispositifs publics d'incitation à l'implantation) (Mucchielli, 1998; Gérardin et Poirot, 2010). La combinaison de ces facteurs est propre à chaque entreprise, ce qui en complexifie sa gestion (Ferrara et Henriot, 2004). (Voir Figure 1 p.25 : Les trois composantes qui influencent les perceptions d'une EMN vis-à-vis de l'attractivité d'un territoire).

L'action publique en la matière est reconnue comme ayant une influence sur le comportement des EMN et le choix du lieu d'implantation d'une EMN ne résulte pas que de considérations internes (Colovic, Mayrhofer, 2008). Dès lors, le choix de localisation des EMN est essentiellement influencé par l'importance de la demande présente 
sur le territoire, le coût des facteurs de production, le niveau de concurrence locale et les politiques locales d'attractivité (Mucchielli, 1998; Mayer et Mucchielli, 1999; Mucchielli et Puech, 2003). Pour autant, la littérature reste discrète sur la manière dont l'action publique locale prend forme et sur les éléments de cette action publique qui permettent d'expliquer la réussite des processus d'implantation des EMN étrangères (Coussi, 2014).

\section{LOGIQUE ET INTERVENTION PUBLIQUES EN MATIÈRE D'ATTRACTIVITÉ TERRITORIALE}

Dans le cadre des dynamiques liant l'EMN et le territoire, il est admis que l'effet de la globalisation des économies tend à gommer l'importance des frontières nationales sans pour autant nier la territorialité des phénomènes économiques; dans une configuration mondiale, l'économie se polarise et renforce l'importance des territoires infranationaux tels que les régions ou les métropoles (Mucchielli et Puech, 2003; McCann et Mudambi, 2005; Rugman et Verbeke, 2009; Narula et Dunning, 2010; Meyer et al., 2011; Rugman et al., 2011). Cette forme économique, tout aussi dispersée qu'intégrée, résultant de forces centrifuge et centripète (Krugman, 1991), constitue un paradoxe qualifié «d'économie d'archipel» (Veltz, 1996). On assiste à une concentration de la croissance mondiale autour de ces grandes métropoles qui constituent des nœuds reliés les uns aux autres et qui dépassent les découpages nationaux (Colletis et Pecqueur, 2005). Dès lors, l'échelon national ne s'impose plus comme médiatrice dans la relation entre global et local (Benko, 1999; McCann et Mudambi, 2005; Rugman et Verbeke, 2009; Rugman et al., 2011). Corollairement, les mouvements de décentralisation dans plusieurs régions du monde impliquent la cohabitation de logiques descendante et ascendante du développement local (Hernandez, 2008). L'attractivité territoriale demeure donc une préoccupation nationale tout en se construisant sur des échelles infranationales.

L'action publique est donc considérée ici comme «multiniveaux", plurielle et protéiforme. Les dispositifs publics d'attractivité recouvrent essentiellement deux modalités, un soutien direct à l'investisseur ou un soutien indirect (Sergot, 2004). Les soutiens directs se matérialisent par des mesures incitatives d'ordres financier (subventions, réductions de charges ou allègement de fiscalité), matériel (disposition d'infrastructures), immatériel (informations ou expertises spécifiques, soutien aux différents réseaux territorialisés d’organisation), (Carré et Levrato, 2009). Le soutien indirect vise à créer des conditions favorables à l'implantation d'entreprises (Marty, 2005). S'il existe des modalités d'incitation directe et indirecte, ces dispositifs peuvent avoir une orientation individuelle ou collective (Carré et Levrato, 2009); l'orientation individuelle vise à apporter un soutien dont l'entreprise peut en bénéficier seule. L'orientation collective, quant à elle, conditionne le soutien par la présence d'un collectif d'entreprises voire d'un collectif à la fois privé et public nécessitant de développer les coopérations entre acteurs d'un territoire (Marty, 2005 : 102).

Cependant, les EMN, conscientes de l'importance que revêt leur choix de localisation (Dunning, 1998), s'efforcent de prendre en compte la dimension régionale de leur localisation et n'hésitent pas à mettre les territoires en concurrence ce qui entraîne souvent des situations de surenchères défavorables pour le développement économique local (Benko, 1999; Carluer, 2005). L'influence des territoires infranationaux, et l'impact de l'action publique locale sur les décisions de localisation des EMN, sont à cet égard d'autant plus prégnants dans le contexte spécifique des pays émergeant du fait de leur forte diversité territoriale (Meyer et Nguyen, 2005; Chan et al., 2010; Ma et al., 2010; Beugelsdijk et Mudambi, 2013; Ma et al., 2013). Aussi, les stratégies de surenchères entre territoires ne garantissent pas le succès et n'infléchissent pas la volatilité des entreprises qui suscitent l'inquiétude des acteurs publics locaux (KrifaSchneider et Baudin, 2007). Crozet et Meyer (2002) soulignent le fait que les entreprises les plus sensibles à ce genre de dispositifs sont finalement les plus volatiles générant ainsi que peu d'externalités positives pour le développement local. Certaines études empiriques en la matière révèlent un lien faible entre les dispositifs publics à dominante pécuniaire et l'implantation de nouvelles entreprises (Houdebine et Schneider, 1997) et d'autres, plus récentes, tendent à considérer que le coût du territoire peut être un déterminant essentiel des décisions de localisation des EMN selon le caractère plus ou moins mobile de l'EMN et selon l'adéquation entre les attributs du territoire et ceux recherchés par l'EMN (Carluer et Foignet, 2012). L'action publique orientée vers des avantages pécuniaires porte en ce sens une vision de court terme qui accentue la concurrence territoriale et accroît le risque lié aux effets de surenchère (Benko, 1999). Néanmoins, contrainte par l'environnement, il est difficile d'imaginer qu'une politique d'attractivité puisse écarter toute forme d'incitation par des avantages pécuniaires au risque de se trouver à la marge des politiques d'attractivité voisines. En revanche, soulignons tout de même que les dispositifs d'attractivité indirecte, à orientation collective, visant à améliorer les infrastructures locales et l'environnement des affaires, influencent significativement le comportement de localisation des EMN (Crozet et Meyer, 2002; Meyer et Nguyen, 2005; Meyer et al., 2011).

C'est ici que réside l'objectif de cette recherche en management public; il s'agit de comprendre dans quelle mesure et comment l'action publique locale peut exercer une influence pour favoriser l'implantation d'EMN étrangères sur son territoire sans pour autant entrer dans des logiques de surenchère. Pour ce faire, l'approche par les ressources est mobilisée comme grille de lecture du choix de localisation et du processus d'implantation des EMN.

\section{LA RENCONTRE PRODUCTIVE ENTRE TERRITOIRE ET EMN : UNE APPROCHE PAR LES RESSOURCES}

Le territoire est ici entendu comme un construit social, à l'intérieur duquel les activités économiques s'encastrent et se coordonnent, porteur de récursivité car il est à la fois le produit et le résultat d'actions collectives. Notre approche du territoire nous invite à prendre en compte l'importance de l'environnement territorial et de l'action publique afférente qui influence le comportement des acteurs et leur rapport au territoire. Dans une telle perspective, l'attractivité territoriale est un enjeu majeur pour les acteurs publics qui se doivent de créer les conditions favorables à l'implantation d'activités économiques (Porter, 2000). Ainsi, en interprétant le processus d'implantation d'une EMN à travers l'échange de ressources, les territoires ne sont 


\begin{tabular}{|c|c|c|c|c|}
\hline \multicolumn{5}{|c|}{$\begin{array}{c}\text { TABLEAU } 1 \\
\text { Les entreprises sélectionnées }\end{array}$} \\
\hline Entreprise & Origine & $\begin{array}{l}\text { Date d'implantation dans } \\
\text { la région de Kalouga }\end{array}$ & $\begin{array}{l}\text { Nombre d'employé dans la } \\
\text { région de Kalouge (2012) }\end{array}$ & Secteur d'activité \\
\hline Entreprise 1 & francaise & 2007 & Environ 3000 & Automobile \\
\hline Entreprise 2 & francaise & 2009 & 157 & Automobile \\
\hline Entreprise 3 & allemande & 2007 & Environ 3500 & Automobile \\
\hline Entreprise 4 & francaise & 2009 & 12 & Automobile \\
\hline Entreprise 5 & allemande & 2010 & 21 & Automobile \\
\hline
\end{tabular}

plus considérés comme de simples supports d'activité et sont analysés "comme des entités socialement construites résultat de processus particuliers de coordination d'acteurs» (Colletis et Pecqueur, $4: 2005$ ).

A l'instar des travaux de Le Gall (2011), nous proposons une approche par les ressources pour une compréhension des tenants de la rencontre productive entre le territoire et l'EMN (Colletis et Pecqueur, 1993). Une telle approche nous permet de caractériser l'orientation de l'action publique et les logiques d'attractivité territoriale afférentes par la mise en perspective des ressources mises à disposition de l'EMN, par les acteurs territoriaux, lors de son processus d'implantation (Couston et al., 2015).

L’approche par les ressources déployée dans le cadre du développement local trouve son origine dans les travaux de Colletis et Pecqueur (1993) et plus largement dans les travaux de l'école de la proximité. Dans une approche penrosienne renouvelée, la ressource dépasse les frontières de l'entreprise (Dyer et Singh, 1998) et dans notre cas, se construit sur un territoire (Colletis et Pecqueur, 1993). Les dispositifs publics d'attractivité territoriale mettent à disposition des entreprises de nombreuses ressources visant à accompagner et faciliter leur implantation (Carluer et Foignet, 2012). Ces ressources, si elles sont multiples, sont généralement regroupées en deux grands ensembles, celles reposant sur des avantages pécuniaires et les autres (Crozet et Meyer, 2002). Par ailleurs, il est possible de classer les ressources selon leur nature tangible ou intangible (Métais, 2004). Si plusieurs classifications de ressource sont disponibles dans la littérature (Penrose, 1959; Barney, 1991; Grant, 1991), nous procéderons à une classification des ressources issue des résultats de la recherche. Ainsi, l'objectif est d'étudier les interactions entre les acteurs territoriaux et l'EMN lors de son implantation, de sorte à identifier les ressources mises à disposition de l'EMN lors de ces interactions. Nous proposons d'intégrer à la grille de lecture des ressources la dichotomie proposée par Colletis et Pecqueur (1993) à savoir les ressources génériques et les ressources spécifiques. Cette dichotomie permet d'apprécier le niveau de territorialité de la ressource et d'en déduire le potentiel d'ancrage territorial de l'EMN. En effet, s'intéresser au caractère spécifique et donc territorial d'une ressource «se présente comme un modèle de développement alternatif (Colletis et al., 1999; Camagni, Maillat et Matteaccioli; 2004; Kebir, 2006) à l'approche classique s'intéressant essentiellement à la dotation factorielle» (François et al., $268: 2013$ ). Ainsi, une ressource faiblement territoriale implique qu'elle soit facilement codifiable, transférable et imitable (ressource générique). Au contraire, une ressource fortement territoriale sera de nature tacite, difficilement imitable et transférable, n'existant qu'à l'état virtuel car elle résulte de l'interaction entre acteurs territorialisés (ressource spécifique) (Colletis et Pecqueur, 2005; Colletis, 2010; François et al., 2014).

La dichotomie «ressource générique» versus «ressource spécifique " engage des trajectoires de développement différenciées (Colletis-Wahl et Perrat, 2004). La trajectoire de développement «basse» est marquée par une faible territorialité des ressources; les ressources mobilisées sont d'ordre générique et sont donc facilement transférables. Dans une logique de prédation, la stratégie de localisation de l'EMN correspond à la recherche du moindre coût et elle sera sensible aux avantages pécuniaires proposés par les acteurs publics. En revanche, la trajectoire de développement "haute» correspond à une forte territorialité des ressources mises à disposition renvoyant alors à des ressources spécifiques qui impliquent l'ancrage territorial de l'EMN et le passage d'une logique de prédation à une logique de construction de ressources (Perrat et Zimmermann, 2005). L'EMN et le territoire sont ici intimement liés puisque l'activation de ressources spécifiques dépendra de l'encastrement de l'EMN dans un tissu de relations territoriales dans la mesure où les ressources spécifiques sont le fruit des interactions entre acteurs territoriaux. La capacité des acteurs du territoire à développer des proximités (géographiques, organisationnelles, institutionnelles), conditionne l'émergence de ressources spécifiques territoriales pour une trajectoire de développement haute (Colletis-Wahl et Perrat, 2004; Torre et Beuret, 2012). Ainsi, l'action publique à orientation collective en matière d'attractivité territoriale poursuit l'objectif d'une trajectoire de développement «haute» dans la mesure où l'accès aux ressources est conditionné par l'insertion dans un collectif et par le développement de coopérations. Néanmoins, le risque majeur de ce type de trajectoire de développement réside dans le phénomène de «myopie » qui peut conduire à des situations 
de «lock-in» en raison d'une approche sur-territorialisée des problèmes productifs (Maskell et Malmberg, 2007).

\section{Méthodologie}

\section{DESIGN DE LA RECHERCHE}

Nous nous intéressons aux interactions entre EMN et acteurs territoriaux lors du processus d'implantation. Notre objectif consiste à étudier la rencontre productive entre acteurs territoriaux et EMN lors de son processus d'implantation par le prisme des ressources échangées afin de mettre en perspective l'action publique en matière d'attractivité. Pour ce faire, nous proposons une étude de cas, celle de la région de Kalouga en Russie qualifiée «d'Eldorado des firmes françaises » notamment par les journalistes russes. Cette étude de cas a été sélectionnée pour plusieurs raisons :

L'effet d'opportunité mettant en exergue les critères définis dans notre corpus théorique comme étant des facteurs d'attractivité territoriale (Krugman, 1993; Gérardin et Poirot, 2010). Le choix de ce critère conduira notre analyse en mettant en avant ce fil conducteur central : la forte symbiose entre les EMN et son environnement. L'environnement russe, notamment le contexte institutionnel, est moins favorable en Russie pour les EMN : «En Russie, la construction des institutions du marché était plus compliquée et même compromise par le rôle de la bureaucratie et des oligarques. Ces groupes dracteurs économiques ont poursuivi leurs intérêts privés, tout en introduisant de nouvelles structures institutionnelles. Dans une certaine mesure, ils ont créé des institutions inefficaces, entraînant l>arbitraire, le libre service et la corruption » (Molz et al., 2009, p. 142). Selon l'article publié en 2012 dans le Courrier de Russie «Kalouga : accompagner les investisseurs de $\mathrm{A}$ à $Я$ », la région de Kalouga offre des conditions parfaites pour des investisseurs étrangers, qui y trouvent notamment une administration locale efficace, portée sur le développement économique et l'attractivité des territoires, et qui sait comment révéler le meilleur du potentiel de sa région.

La taille de l'échantillon et la représentativité des répondants (Miles et Huberman, 1991). Nous avons au départ contacté onze premières entreprises, répondant à nos critères de choix, élaborés suivant notre corpus théorique mobilisé. Ces entreprises ne souhaitaient pas participer à notre étude sur un sujet aussi délicat et politisé en termes d'influences territoriales, nous nous sommes alors adressés au directeur de la CCI. Grâce à son appui, nous avons pu sélectionner, au travers de notre grille de critères de choix, cinq entreprises implantées à Kalouga qui ont accepté de participer à notre étude à la condition de garder leur anonymat. Les personnes choisies pour les entretiens sont des décideurs principaux, centraux pour l'étude de chaque cas, et travaillent sur le site local dans la région de Kalouga.

Un nombre élevé d'entreprises européennes ont fait ce choix par comparaison à d'autres régions : la notion de réputation territoriale est ici importante (Campagne et Pecqueur, 2014). Selon Mme Rozanova aujourd'hui 70 entreprises d'origine européennes se sont installées dans la région qui se caractérise par une forte présence d'expatriés. On compte actuellement 250 Français à Kalouga. En plus des familles installées, il y a également des Français en détachement pour des missions de longues durées. Kalouga est considérée comme la deuxième communauté française de Russie. En 2011, le gouverneur de Kalouga, Anatoliy Artamonov, a été décoré de l〉ordre de chevalier de la Légion d`honneur pour son action efficace en faveur de linvestissement étranger.

Notre méthode de recherche est de nature qualitative et exploratoire, se fondant sur une étude de cas unique enchâssée (Yin, 2003). Notre étude de cas est unique; il s'agit d'un même territoire, celui de la région de Kalouga, en Russie, et enchâssée dans la mesure où notre étude porte sur plusieurs sous-unités d'analyse correspondant aux différentes EMN investiguées (Yin, 2003). Ainsi, cinq EMN, relativement homogènes, ont été choisies; elles appartiennent toutes au même secteur d'activité (industrie automobile), elles sont toutes d'origine européenne, et enfin leur implantation s'est faite sur la même période (20082010). Notre échantillon se compose de trois grandes entreprises (E1, E2, E3) et de deux PME (E4, E5) (tableau 1 p.25). Nous nous sommes focalisés sur les investissements greenfield qui correspondent à la création de sites ex-nihilo plutôt qu'à des opérations de fusion-acquisition ou de restructuration financière. Les opérations de création ex-nihilo (investissement greenfield) et d'extension restent les plus importantes en termes de retombées économiques locales (OCDE, 2008 : 98). (Voir Tableau 1 p.25: Entreprises sélectionnées).

Quant aux sources d'évidence, nous avons privilégié les données primaires, par des entretiens semi-directifs, et les données secondaires. 22 entretiens ont été réalisés au cours de l'année 2011 (tableau 1). Nous avons interrogés des individus présents lors de l'implantation de l'EMN et impliqués dans le processus de décision de localisation. Aussi, pour reconstituer ce processus d'implantation, nous avons opté pour des entretiens semi-directifs de type narratif (Eisenhardt, 1989). Ces entretiens ont été entièrement retranscrits pour une analyse de contenu thématique (Miles et Huberman, 1991) à l'aide du logiciel N'Vivo 10. Nous avons procédé à un codage ouvert et axial qui nous a permis, pour chaque EMN, de recouper les différentes narrations afin d'identifier le rôle des acteurs territoriaux et les ressources mises à disposition de l'EMN. La présence de 5 sous-unités d'analyse conduit à la mise en évidence d'invariants et de codes significatifs en termes de fréquences inter et intra-cas, ce qui nous permet de mettre en exergue les ressources spécifiques territoriales qui caractérisent l'attractivité du territoire de Kalouga perçue par les EMN récemment implantées. Les ressources territoriales ont été codées de manière émergente, sans classification a priori. A contrario, l'appréciation de la territorialité des ressources (qui permet d'identifier la nature générique ou spécifique des ressources) s'est faite selon que ces dernières soient fortement attachées au territoire - car résultant $\mathrm{du}$ jeux des acteurs territoriaux - ou fortement mobiles, et selon qu'elles soient de nature tacite ou codifiable (Le Gall, 2009). (Voir Tableau 2 p.26 : Liste des interviews)

\section{Présentation du TERRAin de Recherche}

Les mouvements de privatisation et la mise en place d'une économie libérale sous le gouvernement des années 1990 ont permis de poursuivre la transition visant le passage d'un système communiste vers une économie de marché qui avait été 
enclenchée par la perestroika et la Glasnost. Ainsi, en 2009, la Russie fait partie des vingt premiers pays d'accueil des IDE et se hisse à la sixième place avec 3,5\% des entrées d'IDE dans le monde (CNUCED, 2010). Par ailleurs, la Russie, par son étendue géographique et ses disparités économiques et politiques entre régions, nous conduit à avancer le rôle des acteurs publics locaux et leurs politiques d'attractivité territoriale comme des facteurs déterminants dans le choix de localisation des EMN qui en explique pour partie l'attractivité du territoire étudié.

Située à 70 kilomètres au sud-ouest de Moscou, avec plus d'un million d'habitants, la région de Kalouga attire un nombre croissant d'EMN européennes depuis 2006. En 2011, la région enregistre 74 accords officiels signés et 20 accords en cours de négociation avec des entreprises étrangères de différentes nationalités. En 2010, Kalouga est devenue la première région russe en volume d'IDE avec 1,25 milliards de dollars. Il s'agit d'une région attractive qui a su mettre en œuvre une stratégie d'attractivité territoriale pour assurer le développement économique de son territoire. Dans les années 1990 les pouvoirs publics locaux acquièrent leur autonomie par un processus de décentralisation et se voient confier le développement de leur territoire, à l'époque caractérisé par une économie agricole et la présence d'une seule industrie d'armement. L'attractivité actuelle de la région s'explique notamment par la mobilisation des pouvoirs publics locaux pour mettre en œuvre une stratégie territoriale orientée vers un développement exogène visant l'implantation d'entreprises exclusivement étrangères.

La mise en ouvre d'un projet territorial a favorisé le développement des réseaux routiers, de la viabilisation des terrains, de l'obtention rapide des permis de construire, et la réflexion autour d'avantages fiscaux intéressants par rapport aux autres régions russes. Enfin, la région dynamise aussi sa politique en faveur des familles d'expatriés. Toutes ces actions ont été mises en place par les acteurs de la gouvernance locale sous l'impulsion d'un fort leadership politique, porté notamment par un gouverneur entreprenant. L'objectif poursuivi par les acteurs publics territoriaux repose ainsi sur la volonté de «construire une relation privilégiée et offrir un soutien personnel».

\section{Résultats : Les acteurs territoriaux et les pratiques de management territorial comme déterminants des décisions de localisation dans la région de Kaluga.}

\section{LES MOMENTS CLÉS DU PROCESSUS D'IMPLANTATION DES 5 EMN INVESTIGUÉES}

Pour les 5 EMN investiguées, trois moments déterminants dans le processus d'implantation des EMN s'illustrent. Le premier renvoie à la rencontre entre l'EMN et le territoire d'implanta-

\begin{tabular}{|c|c|c|c|}
\hline \multicolumn{4}{|c|}{$\begin{array}{l}\text { TABLEAU } 2 \\
\text { Liste des interviews, source auteurs }\end{array}$} \\
\hline Entreprise & Répondant & Département & Durée de l'entretien \\
\hline \multirow[t]{5}{*}{ E1 } & R1 & Communication & $1 \mathrm{~h} 48$ \\
\hline & R2 & Administration & $1 \mathrm{H} 12$ \\
\hline & R3 & Administration & $1 \mathrm{~h} 00$ \\
\hline & R4 & Production & $1 \mathrm{~h} 00$ \\
\hline & R5 & Logistique & $1 \mathrm{~h} 20$ \\
\hline \multirow[t]{3}{*}{ E2 } & R1 & Administration & $2 \mathrm{~h} 38$ \\
\hline & R2 & Marketing & $1 \mathrm{~h} 15$ \\
\hline & R3 & Production & $1 \mathrm{~h} 00$ \\
\hline \multirow[t]{5}{*}{ E3 } & R1 & Administration & $1 \mathrm{~h} 20$ \\
\hline & R2 & Administration & 1h45 \\
\hline & R3 & Marketing & $1 \mathrm{H} 15$ \\
\hline & R4 & Production & $1 \mathrm{H} 50$ \\
\hline & R5 & Production & $1 \mathrm{~h} 20$ \\
\hline \multirow[t]{4}{*}{ E4 } & R1 & Administration & $1 \mathrm{~h} 45$ \\
\hline & R2 & Administration & $1 \mathrm{H} 20$ \\
\hline & R3 & Marketing & $1 \mathrm{H} 50$ \\
\hline & R4 & Communication & $2 \mathrm{H} 35$ \\
\hline \multirow[t]{5}{*}{ E5 } & R1 & Administration & $1 \mathrm{~h} 20$ \\
\hline & R2 & Administration & $1 \mathrm{H} 34$ \\
\hline & R3 & Production & $1 \mathrm{H} 45$ \\
\hline & R4 & Logistique & $1 \mathrm{H} 00$ \\
\hline & R5 & Marketing & $1 \mathrm{H} 35$ \\
\hline
\end{tabular}


tion : «Les relations avec la région ont commencé en 2005 quand nous avons rencontré les représentants de la région à Moscou, après nous avons été invités à visiter la région...» (R2, E2). A ce moment précis, les EMN n'ont pas encore arrêté leur choix de localisation au niveau infranational. Si celui-ci répond à un processus séquentiel comportant le choix du continent, du pays, d`une région et enfin d`un site particulier (Crozet et Mayer, 2002), cette rencontre a particulièrement trait au choix de la région. Par conséquent, le choix du pays est arrêté : "Au début nous avons choisi trois régions... » (R2, E1). Il s'agit ensuite de préciser la localisation du site ce qui constitue une deuxième phase dans le processus d'implantation. Cette phase vise à approfondir les négociations afin de fixer les conditions d'accès au territoire et au site d'exploitation : «Après nous être renseignés sur la région auprès de nos partenaires on nous a proposé plusieurs sites, nous en avons choisi un localisé pas loin de la capitale régionale» (R1, E4). La dernière phase est celle de la contractualisation entre les acteurs publics locaux et l'EMN : «La signature du contrat avec la région est devenue la phase finale (...)»(R2, E2). A la suite de ce dernier moment, l'EMN entre dans sa phase d'exploitation et investie les lieux : "Après la signature du contrat les choses sérieuses ont commencé : il fallait organiser les travaux, nous installer quelques part en attendant» (R3, E3). Ainsi, nous obtenons trois phases dans le processus d'implantation dont découle, pour chaque phase, des interactions et des échanges de ressources spécifiques.

\section{LES INTERACTIONS ENTRE ACTEURS TERRITORIAUX ET EMN}

Parmi les acteurs territoriaux principalement cités par les personnes interrogées, nous distinguons en premier lieu les acteurs publics dont la collectivité territoriale "régionale», l'organisation chargée de la représentation de la région de Kalouga à Moscou, la collectivité territoriale «locale», la CCI, et enfin l'agence de développement économique. Concernant les acteurs privés, on observe le rôle important des membres du cluster local, du fond d'investissement et enfin des clubs et des associations d'entreprises.

Dans la première étape, liée à la rencontre, les futurs investisseurs passent d'abord par une organisation chargée de la représentation de la région de Kalouga à Moscou (16 répondants sur 22) ou par l'agence de développement économique locale (14 répondants sur 22). L’organisation chargée de la représentation de la région de Kalouga à Moscou, appelée par les habitants de Kalouga «mini-ambassade», participe activement dans la vie économique du pays et organise les foires et salons professionnels : «... j’ai croisé plusieurs fois les représentants de la région faisant partie des personnes qui travaillent pour la mini-ambassade de la région, on a échangé des idées et on a été invité à visiter la région» (E4, R2). Récemment créée, l'agence de développement économique devient un acteur central de ce réseau territorial. Un des répondants de l'E5 implantée dans la région en 2010 caractérise cette rencontre de la façon suivante : «Pour notre entreprise, c'est l'acteur le plus important, toutes nos communications passaient par elle..». Les répondants soulignent également, de manière significative (17 répondants sur 22), l'implication du gouverneur de la région et de son équipe. Le directeur de la communication de l'entreprise E1 affirme que : "Le gouverneur et son équipe sont toujours à notre écoute, toujours disponibles. Pour n'importe quelle demande, on a eu une réponse en 48 heures. Du jamais vu en Russie». Globalement, les acteurs privés ne sont pas présents en revanche dans cette phase décisionnelle.

La deuxième étape, liée à la phase de négociation est fortement marquée par une intervention de la collectivité territoriale locale (18 répondants sur 22) qui participe de plus en plus activement aux négociations et par celle de l'agence de développement (14 répondants sur 22) qui accompagne, dans toutes les démarches, les investisseurs sur le territoire. Comme pour la première étape, le rôle de l'équipe du gouverneur est très important (17 répondants sur 22$)$ : «Les négociations ont duré pendant plusieurs mois, on a senti une forte motivation de l'équipe régionale et le soutien du gouverneur même» (R3, E2). Dans cette étape, les entreprises rencontrent les CCI (11 répondants sur 22) et également plusieurs acteurs privés comme des associations d'entreprises (8 répondants sur 22) et le fonds d'investissement ( 6 répondants sur 22). Les acteurs du cluster local renforcent leur influence (14 répondants sur 22). La dernière phase de contractualisation correspond au renforcement de l'intervention des acteurs publics : les acteurs de la collectivité territoriale locale (15 répondants sur 22) et la CCI (9 répondants sur 22) entrent en interaction avec les EMN afin de faciliter leur implantation sur le territoire. Ici, la collectivité territoriale régionale (17 répondants sur 22) et l'agence de développement économique (15 répondants sur 22) conservent un rôle central. Finalement, les échelons régionaux interviennent à chaque phase du processus décisionnel. Nous observons un affaiblissement du rôle des acteurs privés (cluster local : 6 répondants sur 22; fonds d'investissement : 3 répondants sur 22; clubs et associations d'entreprises : 3 répondants sur 22) : «Nos principaux partenaires dans la région sont les pouvoirs publics locaux» (R2, E4). Précisons que l'étude de cas met en évidence des relations différenciées avec les acteurs territoriaux selon la

TABLEAU 3

Liste des interviews en 2016 , source auteurs

\begin{tabular}{|l|l|l|l|}
\hline Entreprise & Répondant & Département & Durée de l'entretien \\
\hline E1 & R1 & Communication & 20 minutes \\
\hline E2 & R1 & Administration & 25 minutes \\
\hline E3 & R1 & Marketing & 35 minute \\
\hline E4 & R1 & Administration & 40 minutes \\
\hline E5 & R1 & Administration & 20 minutes \\
\hline
\end{tabular}


taille de l'entreprise considérée. Nous avons investigué cinq EMN dont trois grandes entreprises (E1, E2, E3) et deux PME $(\mathrm{E} 4, \mathrm{E} 5)$. Les acteurs régionaux jouent un rôle plus important que les acteurs locaux dans le processus d'implantation d'une grande entreprise. En revanche, les EMN de petite taille ont des liens plus forts avec les acteurs locaux. Pour les PME, le développement des liens sociaux leur permet de mobiliser le capital social du territoire pour la réussite de leur entreprise (Johannisson et al., 2002).

\section{LES RESSOURCES MISES À DISPOSITION DES EMN PAR LES ACTEURS TERRITORIAUX}

Lors de la première rencontre, les ressources recherchées par les EMN sont principalement de nature intangible et émanent des acteurs publics. Ces derniers fournissent des informations sur leur territoire et en transmettent une image positive, mettant en avant ses atouts : "L'originalité de l'implantation en Russie vient tout d'abord d'une forte influence des pouvoirs publics locaux (...) dans la région de Kalouga, les collectivités territoriales régionales et l'agence de développent économique ont mis à notre disposition des ressources relationnelles" (R1, E4). "On entend beaucoup parler de la Région. Plusieurs articles de presse sont apparus dans les revues et magasines locaux» (R5, E1).

Les entreprises cherchent à réduire leurs incertitudes et s'orientent vers les acteurs publics afin d'acquérir des connaissances sur le territoire : "S’implanter en Russie représentait un grand risque pour notre entreprise (...) nous avons cherché avant tout à collaborer avec les acteurs publics locaux» (R1, $\mathrm{E} 2)$. «On a souvent croisé les représentants de la région au salons professionnels en Russie et même à l'étranger, tout le monde en parle à Moscou» (R4, E2).

Dans la phase de négociation et de contractualisation, la collectivité territoriale locale entre en scène pour fixer les conditions d'accès au territoire. Les acteurs publics et les EMN négocient les ressources matérielles et financières qui seront mises à disposition à la suite de la phase de contractualisation. Il s'agit en ce sens de ressources tangibles mises à disposition des EMN par les acteurs publics locaux: «Pendant les négociations nous avons précisé les éléments matériels comme par exemple la location temporaire des bureaux en attendant la fin des travaux de notre usine (...) les conditions d'un crédit éventuel» (R1, E5). Pour autant, durant cette phase, les EMN sont toujours à la recherche d'informations. Les acteurs publics ont toujours le rôle de pivot et les orientent également vers les acteurs privés, par l'intermédiaire du cluster local et des associations d'entreprises : "L'agence de développement nous a orienté dans le choix de nos futures partenaires régionaux. On a rencontré une association locale grâce à la CCI» (R2, E3).

$\mathrm{Au}$ fur et à mesure que l'on avance dans le processus d'implantation, les EMN recherchent des informations plus précises en lien direct avec leur future exploitation : "Nous avons eu besoin d'information sur la qualité et les disponibilités du logement à Kalouga, car louer un appartement en Russie est très difficile» (R1, E1). Ici, les résultats soulignent l'importance des pratiques de management territorial et le rôle des acteurs publics dans les décisions de localisation des EMN.
LA CRISE UKRAINIENNE, LES SANCTIONS CONTRE LA RUSSIE ET LEURS IMPACTS SUR LES ENTREPRISES ÉTUDIÉES

La situation récente de 2015 avec la crise Ukrainienne et les sanctions contre la Russie pouvaient avoir un impact non négligeable sur la présence et la performance de ces entreprises. Nous avons donc décidé de reprendre contact avec elles afin de savoir si ces dernières restent encore ancrées à Kalouga et comment la crise récente interagit avec l`attractivité du territoire. Nous avons donc réalisé cinq entretiens téléphoniques en janvier 2016 avec les représentants de cinq entreprises (voir Tableau 3 p.27 : Liste des interviews en 2016).

Les entretiens téléphoniques ont montré que les cinq entreprises souhaitent rester présentes dans la région. La situation politique n'a pas eu d'impact négatif sur la performance des entreprises (les chiffres d'affaires continuent de progresser). L'entreprise E3 a planifié l'ouverture d'une deuxième unité de production en 2017. L'entreprise E2 a créé 120 emplois supplémentaires dans la région en 2015. Aucune entreprise ne planifie de fermer les unités de production et de quitter la région.

Après avoir analysé les articles de presse et les discours téléphoniques des personnes interrogées, nous avons appris que la région continuait d'accueillir d'autres entreprises. Par exemple, le groupe pharmaceutique danois Novo Nordisk a ouvert le 15 avril dernier sa première usine sur le territoire russe, dans la région de Kalouga. L'usine fabriquera de l'insuline destinée aux 10 millions de diabétiques russes. La région continue sa croissance avec l'ouverture d'un aéroport à Grabtsevo qui a inauguré ses pistes le 16 juin 2015. Enfin en 2015, le responsable de la région de Kalouga, Anatoli Artamonov, a été reconnu «gouverneur le plus performant» de Russie dans une étude réalisée par l'ONG russe Fondation du développement de la société civile.

\section{Discussion : Des ressources territoriales vers une trajectoire de développement «haute»?}

\section{DES RESSOURCES DE NATURES IDÉELLE, INFORMATIONNELLE ET RELATIONNELLE}

Quelle que soit la phase du processus d'implantation des cinq EMN, l'incertitude constitue l'élément qui concentre l'attention des décideurs et les efforts déployés visent à la réduire. Notons d'abord que les EMN investiguées appartiennent au même secteur d'activité et ont la même origine géographique. Aussi, ces critères ont guidé leur choix de localisation en se rapprochant des EMN déjà implantées afin d'acquérir des connaissances à travers les expériences d'autres EMN qu'elles considèrent «culturellement » proches (Johanson et Valhne, 1990; Mayrhofer et Urban, 2011). Dans une approche néo-institutionnelle, la présence d'EMN qui leur ressemblent, comme facteur explicatif de la localisation, renvoie à une logique d'isomorphisme mimétique qui se concrétise par la préférence pour «des aires géographiques familières" (Sergot, 2007 : 112). Dès lors, à l'intérieur de la région, les EMN recherchent une localisation proche des autres structures déjà implantées.

Notons également que les acteurs publics de Kaluga ont pleinement conscience de l'aversion au risque des EMN. Les acteurs interrogés ont souligné le rôle de l'agence de développement de la région de Kaluga. Or, cette agence a été créée sur 
le modèle français et résulte d'une stratégie des acteurs publics locaux d'attirer des EMN européennes en réduisant la distance administrative (Ghemawat, 2001; Angué et Mayrhofer, 2010; Mayrhofer et Urban, 2011) par la création d'un environnement administratif familier. En effet, la création de cette agence permet de construire un environnement institutionnel marqué par le partage de normes et d'un référentiel commun qui facilite le processus d'implantation et favorise l'attractivité du territoire.

De manière générale, le rôle des acteurs publics et les pratiques de management territorial ont été déterminants dans les décisions de localisation des cinq EMN. Par l'étude des interactions entre acteurs territoriaux et EMN, les résultats révèlent que les ressources mises à disposition des EMN se rapprochent des classifications issues de la littérature (Grant, 1991; Hall, 1992; Métais, 2004). Nous distinguons des ressources tangibles telles que des ressources matérielle et financière, mais surtout intangibles. En effet, les résultats indiquent que ce sont les ressources de nature intangible qui ont été déterminantes dans les décisions de localisation des EMN investiguées, notamment celles de nature informationnelle correspondant à la qualité des informations données aux EMN par les acteurs territoriaux. Les ressources de nature idéelle sont liées à l'image du territoire et à sa promotion auprès des EMN. La démarche des acteurs publics correspond à une logique de marketing territorial; l'enjeu consiste à rendre le territoire attrayant pour les EMN (Noisette et Valérugo, 2010). Enfin, les ressources relationnelles sont liées à la capacité des acteurs territoriaux à socialiser les EMN et à les insérer dans les réseaux locaux pertinents pour ces dernières. En effet, nous avons pu constater les efforts déployés par les acteurs publics locaux pour socialiser les EMN sur le territoire en les mettant notamment en relation avec les acteurs du cluster local. Nous retrouvons ici l'idée selon laquelle l'action publique locale tend à soutenir l'activité industrielle dominante de son territoire en créant un environnement favorable à l'implantation d'EMN étrangères, similaires à celles déjà présentes (Maskell et Malmberg, 2007; Dunning, 1998; Narula et Dunning, 2010; Nell et al., 2011).

Précisons que les ressources mises à disposition, si elles concernent le territoire de Kalouga, ne lui sont pas spécifiques lorsqu'elles sont prises séparément. Dès la première rencontre, les EMN bénéficient de ressources qu'elles auraient aussi bien pu trouver sur un autre territoire, face à une autre configuration de l'action publique territoriale. Néanmoins, la qualité des ressources relationnelles et l'expertise des acteurs publics régionaux, leur capacité à collaborer et se montrer disponibles, tendent à nuancer nos propos; l'action collective nécessite la confiance, la réciprocité et le partage qui ne sont pas propres à tous les systèmes de gouvernance territoriale du pays. Il s'agit d'une capacité inter-organisationnelle propre aux acteurs publics du territoire de Kalouga. En ce sens, la combinaison et la coordination des ressources informationnelles, idéelles et relationnelles, entre acteurs publics, se transforment en compétence spécifique territoriale liée à la qualité du système de gouvernance territoriale.

Il s'agit donc d'une compétence clé propre à la gouvernance territoriale de Kaluga entendue au sens de Prahalad et Hamel (1990 : 84) comme une capacité «à démultiplier ses ressources en les combinant». Ainsi, à travers la combinaison des ressources idéelles, informationnelles et relationnelles, les acteurs publics parviennent à construire des ressources fortement territoriales qui caractérisent l'attractivité du territoire. La territorialité de ces ressources est liée à leur nature tacite et faiblement codifiable car résultant du jeu des acteurs territoriaux (Le Gall, 2009, 2011).

\section{REMISE EN QUESTION DE LA VARIABLE PRIX COMME DÉTERMINANT DES DÉCISIONS DE LOCALISATION}

Pour rendre leur territoire attractif, les acteurs publics locaux ont mis en œuvre un projet territorial leur permettant de développer les conditions d'exploitation d'une activité économique et plus largement les conditions de vie sur le territoire. En ce sens, le projet territorial constitue bien un facteur d'attractivité (Crozet et Meyer, 2002). Dès lors, les déterminants classiques du choix de localisation liés à un simple arbitrage de coûts laissent place à des déterminants tels que l>environnement des affaires, la qualité des infrastructures, la présence de cluster, une position nodale spécifique de la région dans un tissu étendu de relations économiques et l>accès à des compétences locales. En effet, contrairement à d'autres travaux qui soulignent la variable prix et l'importance de son optimisation pour favoriser l'implantation d'EMN étrangères (Carluer et Foignet, 2012), l'étude de cas menée indique que les avantages pécuniaires proposés par les dispositifs publics d'attractivité n'ont été discutés que lors de la phase de négociation et de contractualisation, lorsque les EMN ont d'ores et déjà fait leur choix régional. Cette observation tend à montrer une faible efficacité de ces dispositifs comparativement à la capacité des acteurs publics à entretenir un tissu de relation et à faire profiter de leur expertise territoriale.

Ainsi, les politiques d'attractivité territoriale classiques, orientées vers de simples subventions, des allègements de charges et de taxes, ne suffisent plus. Cette orientation individuelle avec un soutien direct à l'entreprise est non seulement dangereuse, car entraînant des effets de surenchères (Benko, 1999), mais elle ne convient plus aux besoins des entreprises investiguées. Il en va alors du nécessaire ciblage des EMN par les manageurs territoriaux qui doivent s'assurer de la cohérence entre l'activité de l'EMN et le projet territorial afin d'opérer un alignement stratégique pour une relation gagnant-gagnant. C'est ce que Kalouga a entrepris avec la volonté d'attirer des EMN européennes issues du secteur automobile. Dès lors, la gestion de l'attractivité territoriale s'avère être une activité stratégique qui nécessite une vision sur le long terme et des processus managériaux intégratifs pour mettre en phase le développement local et l'activité des EMN (Narula et Dunning, 2010).

\section{DES DISPOSITIFS PUBLICS D'ATTRACTIVITÉ À ORIENTATION COLLECTIVE ET UNE LOGIQUE D'ACTION PROACTIVE POUR UNE TRAJECTOIRE DE DÉVELOPPEMENT «HAUTE»}

Les dispositifs d'attractivité s'orientent vers une dimension collective (Maskell et Malmberg, 2007) articulant soutien direct et indirect (Léon et Sauvin, 2010). Par le projet territorial et des processus intégratifs de management de l'attractivité, les dispositifs d'attractivité s'orientent de plus en plus vers le collectif impliquant le passage d'une logique réactive à une logique proactive où l'on conditionne l'accès aux ressources territoriales par l'insertion dans un collectif d'acteurs, créant ainsi des interdépendances. 
Dans la relation "territoire-EMN", la nature des liens dépend en partie "de l'ouverture des acteurs locaux vis-à-vis des investisseurs étrangers» (Colovic et Mayrhofer, 54 : 2011). En ce sens, dans notre cas, la capacité relationnelle des acteurs publics locaux a montré, à bien des égards, combien elle était importante pour convaincre les EMN et accompagner leur processus dimplantation. Ainsi, le territoire de Kalouga semble prendre une trajectoire de développement «haute» (ColletisWahl et Perrat, 2004), par rapport aux autres territoires russes, en développant des ressources territoriales spécifiques d'essence idéelle, informationnelle et relationnelle. Ces dernières se matérialisent par l'effort consenti par les acteurs publics locaux pour construire une relation avec les EMN, par leur implication dans le processus d'implantation, leurs compétences en termes de coordination et de gouvernance territoriale, et par l'orientation collective des dispositifs publics d'attractivité. Néanmoins, cette trajectoire ne pourra être appréciée que sur le long terme selon que ces EMN européennes s'orientent ou non vers l'ancrage territorial (Perrat et Zimmerman, 2003). Aussi, notons que les dispositifs d'attractivité à orientation collective qui favorisent l'ancrage territorial en développant des interdépendances peuvent également mener à des situations de lock-in dues à une trop forte spécialisation du territoire (Maskell et Malmberg, 2007). Il convient alors de penser la combinaison de trajectoires de développement «haute» avec la diversité des activités industrielles dans les politiques d'attractivité territoriale.

\section{En conclusion}

La question de l'attractivité d'un territoire et de son pilotage constitue un défi majeur pour l'action publique territoriale, notamment dans un environnement économique de plus en plus globalisé, impliquant un éclatement mondial des maillons d'une même chaîne de valeur (Gereffi et Lee, 2012). Nous avons investigué le cas de la région de Kalouga en Russie à travers l'analyse de cinq processus d'implantation d'EMN étrangères d'origine européenne. Les apports de la recherche menée résident d'abord dans la confirmation empirique du lien entre action publique locale et décisions de localisation des EMN; les résultats soulignent le poids du rôle des manageurs publics territoriaux dans le choix de localisation des EMN. Ensuite, les résultats de l'étude permettent de compléter la littérature en identifiant les éléments de cette action publique qui apparaissent déterminants. En effet, contrairement à l'importance donnée dans la littérature à la variable prix pour expliquer les décisions de localisation, les résultats indiquent que les ressources déterminantes mises à disposition des EMN par l'action publique ne sont pas de nature financière. Il s'agit de ressources de natures idéelle, informationnelle et relationnelle, mises à disposition par les acteurs publics, qui ont été déterminantes dans le processus d'implantation des EMN et ont permis de réduire l'incertitude qui entoure les décisions de localisation à l'étranger. Par ailleurs, cet article met en lumière un cas original qui reste peu investigué, celui de la région de Kaluga en Russie.

Ainsi, la localisation et l'exploitation d'une activité sur un territoire étranger ne peuvent se faire sans intervention publique. Par ailleurs, cette dernière ne s'arrête pas à la fourniture de biens et services; les acteurs publics sont pour les EMN de puissants réducteurs d'incertitude, qui, non seulement facilitent mais aussi accompagnent le processus d'implantation. Ainsi, bien avant l'exploitation de l'activité économique sur le territoire étranger, l'EMN est encastrée dans un tissu de relations territoriales. Charge aux acteurs publics de gérer ces relations et d'orienter le plus possible le comportement de l'EMN vers un ancrage territorial, par l'insertion dans un collectif. Dès lors, la qualité des pratiques de management territorial devient un facteur d'attractivité territoriale.

Pour autant, bien que cette recherche apporte un éclairage spécifique sur le lien entre action publique locale et implantation des EMN étrangères dans cette région Russe, nous pouvons relever plusieurs limites. D'abord, la taille de l'échantillon et le contexte spécifique du territoire investigué qui ne permettent pas de généraliser les résultats apportés. Ensuite, nous avons interrogé les acteurs des EMN impliqués dans les décisions de localisation de manière rétrospective ce qui comporte un risque de rationalisation a posteriori (Le Gall, 2009). Par ailleurs, la spécificité du pays étudié, compte tenu notamment de la forte incertitude politique et administrative de la Russie, surtout depuis la crise Ukrainienne, il est possible que les entreprises qui décident de s'y implanter soient principalement sensibles à trouver une région qui minimise les couts aujourd'hui. Il y aurait donc une nécessité, dans les années à venir, de revenir évaluer ce terrain pour comprendre si cette variable que nous avions décrite comme mineure lors de notre investigation de terrain, a évolué dans les nouvelles réalités géopolitiques.

Quatre voies de recherche complémentaires sont envisageables. Tout d'abord intégrer à notre modèle d'analyse une approche par la théorie des coûts de transaction et l'utilisation du concept de choix spécifiques, afin de donner un éclairage nouveau au corpus théorique mobilisé. Ensuite, il serait intéressant de mener une étude comparative en étudiant des cas d'échec et en choisissant notamment d'autres régions de Russie, a priori moins attractives. Il convient également de multiplier les études de cas et d'intégrer les perceptions des acteurs publics locaux de sorte à enrichir les résultats et à apporter plus de finesse dans l'analyse de l'action publique locale par le truchement des différents flux de perception. Enfin, il serait intéressant d'observer l'évolution de la trajectoire de développement du territoire et d'identifier la manière dont l'action publique parvient à éviter les risques de lock-in spatial en gérant la dualité entre l'ancrage territorial des EMN et l'ouverture du territoire d'une part, et d'autre part la dualité entre trajectoire de développement «haute» et diversité du tissu industriel local.

\section{Bibliographie}

Andersson U, Forsgren M, Holm U, (2002), «The strategic impact of external networks : subsidiary performance and competence development in the multinational corporation", Strategic Management Journal, vol. 23, n¹1, p. 979-996.

Angué K, Mayrhofer U, (2010), «Le modèle d’Uppsala remis en question : une analyse des accords de coopération noués dans les marchés émergents ", Management international, vol. 15, $\mathrm{n}^{\circ} 1$, p. 33-46.

BARney J.B, (1991), «Firm Resources and Sustained Competitive Advantage», Journal of Management, vol.17, n¹, p. 99-120. 
Benko G, (1999), «Marketing et Territoire», dans Fontan, J.M.; Klein, J.L; Tremblay, D.G. (dir.). Entre les métropolisations et le village global, Presse de l'Université du Québec, p. 79-122.

Beugelsdijk S., Mudambi R. (2013), «MNEs as border-crossing multi-location enterprises : The role of discontinuities in geographic space», Journal of International Business Studies, vol. $44, \mathrm{n}^{\circ} 5$, p. 413-426.

CAmpagne P, Pecqueur B, (2014), «Le Développement territorial : Une réponse émergente à la mondialisation», Edition charles leopold mayer

Carluer F, Foignet J-J, (2012), «Les projets d'investissement internationalement mobiles : recours au yield management pour les politiques territoriales d'attractivité? », Management international/International Management/Gestiòn Internacional, vol. $17, \mathrm{n}^{\circ} 1$, p. 39-56.

CARluer F, (2005), «Entreprise-réseau versus réseau d'entreprises territorialisé : vers une matrice d'analyse stratégique», Cahier de recherche $n^{\circ} 31$ de l'Ecole de Management de Normandie, p.1-17.

Carré D, Levratto N, (2009), «Politique industrielle et PME : nouvelle politique et nouveaux outils?", Revue d'économie industrielle, $\mathrm{n}^{\circ} 126$, p.9-30.

Chan C. M., Makino S., Isobe T. (2010), «Does subnational region matter? Foreign affiliate performance in the United States and China", Strategic Management Journal, vol.31, $\mathrm{n}^{\circ} 11, \mathrm{p}$. 1226-1243.

Colletis G, (2010), «Co-évolution des territoires et de la technologie : une perspective institutionnaliste», Revue d'économie régionale et urbaine, $\mathrm{n}^{\circ} 2$, p. 235-249.

Colletis G, Pecqueur B, (1993), «Intégration des espaces et quasi-intégration des firmes : vers de nouvelles rencontres productives? ", Revue d'Economie Régionale et Urbaine, $\mathrm{n}^{\circ} 3$, p.489-508.

Colletis G, Pecqueur B, (2005), «Révélation de ressources spécifiques et coordination située", Revue Economie et Institution, $\mathrm{n}^{\circ}$ 6-7, p.1-16.

Colletis-Wahl K, Perrat J, (2004), «Proximités et dynamiques spatiales», dans Colletis, G.; Pecqueur, B. (dir.) Economie de proximités, Hermès-Lavoisier, Paris.

Colovic A, Mayrhofer U, (2008), «Les stratégies de localisation des firmes multinationales Une analyse du secteur automobile», Revue française de gestion, $\mathrm{n}^{\circ} 184, \mathrm{p} .151-165$.

Colovic A, Mayrhofer U, (2011), «La reconfiguration de l'espace mondial et les stratégies de localisation des firmes multinationales », Management International, vol.16, $\mathrm{n}^{\circ} 1$, p.51-59.

Coussi O, (2014), «Management public de projets d'investissement direct étrangers et intelligence économique territoriale», Gestion et management public, vol. $3, \mathrm{n}^{\circ} 2$, p. 53-74.

Couston A, Fouchet R, Keramidas O, Larat F, (2015), "Les Stratégies d'alliance à l'international des grandes écoles d'administration et des universités françaises et des écoles de service public : l'apport de l'analyse en termes de coopétition". IASIA Colloque annuel, Paris, 6 au 10 juillet 2015.

Crozet M, Mayer T, (2002), «Entre le global et le local, quelle localisation pour les entreprises? Les déterminants de la localisation des firmes ", Les Cahiers Français n³09, p.1-11.
DUNNING J.H, (1998), «Location and the multinational enterprise: A neglected factor?», Journal of International Business Studies, vol. $29, \mathrm{n}^{\circ} 1$, p. 45-66.

EISEnhaRDT K.M, (1989), «Building Theories from Case Study Research », Academy of Management Review, vol.14, $\mathrm{n}^{\circ} 4$, p.532-550.

Ferrara L, Henriot A, (2004), «La localisation des entreprises industrielles : comment apprécier l'attractivité des territoires?", Economie internationale, ${ }^{\circ} 99$, p. 91-111.

François H, Hirczak M, SEnil N, (2013), «De la ressource à la trajectoire : quelles stratégies de développement territorial?», Economie, Géographie, Société, n¹5, p. 267-284.

Gérardin H, Poirot J, (2010), «L'attractivité des territoires : un concept multidimensionnel», Mondes en Développement, vol.38, $\mathrm{n}^{\circ} 149, \mathrm{p} .27-41$.

Gereffi G, LeE J, (2012), «Why the world suddenly cares about global supply chains", Journal of Supply Chain Management, vol. $48, \mathrm{n}^{\circ} 3$, p. 24-32.

Ghemawat P, (2001), «Distance Still Maters. The Hard Reality of Global Expansion», Harvard Business Review, vol.79, nº, p.137-147.

Girod-SÉville M, Perret V, (1999), «Fondements épistémologiques de la recherche», in R- A Thiétart (Ed.), Méthodes de recherche en management. Paris : Dunod.

Grant R.M, (1991), «The Resource-Based Theory of Competitive Advantage : Implications for Strategy Formulation », California Management Review, vol.33, $\mathrm{n}^{\circ} 3$, p.114-135.

HALL R, (1992), «The strategic analysis of intangible resources», Strategic Management Journal, $\mathrm{n}^{\circ} 13$, p.135-144.

Hatem F, (2005), «Attractivité du territoire : de la théorie à la pratique", L'industrie en France et la mondialisation, Service des études et des statistiques industrielles.

Hernandez S, (2008), « Paradoxes et management stratégique des territoires : étude comparée de métropoles européennes ", Revue Vie \& Sciences Economiques, n¹78, p. 54-75.

Houdebine M, Schneider J.L, (1997), «Mesurer linfluence de la fiscalité sur la localisation des entreprises», Document de l'INSEE.

JAYET H, (1993), «Territoire et concurrence territoriale», Revue d'économie régionale et urbaine, ${ }^{\circ} 1$, p. 55-75.

Jensen P. D. Ø., Pedersen T. (2011), «the Economic Geography of Offshoring : the Fit Between Activities and Local Context», Journal of Management Studies, vol. 48, n², p. 352-372.

Johannisson B, Ramirez-Pasillas M, Karlsson G, (2002), "The institutional embeddedness of local inter-firm networks: a leverage for business creation", Entrepreneurship and Regional Development, vol.14, p.297-315.

Johanson J, Vahlne J.E, (1990), The mechanism of internationalization, International Marketing Review, vol.7, n4, p.12-24.

Keramidas O, (2012), «Les trajectoires d'équité dans les processus décisionnels des organisations publiques», "Management International», vol.16, n02, pp.93-112, mai.

Kim J., Aguilera R. (2015), «Foreign Location Choice : Review and Extensions», International Journal of Management Reviews, vol. 00, p. 1-27.

KRUGMAN P, (1991), «Increasing returns and economic geography», Journal of Political Economy, vol.99, n³, p. 483-499. 
Krugman P, (1993), «First nature, second nature, and metropolitan location", Journal of Regional Science, vol.33, n², p. 129-144.

Krugman P, (1995), Development, Geography and Economic Theory, MIT Press, Cambridge.

LE GAll S, (2009), "Les stratégies de localisation des entreprises multinationales et leurs rapports au territoire», Thèse de Doctorat en Sciences de Gestion, Université de Caen Basse-Normandie.

LE GALL S, (2011), «Les stratégies de localisation des firmes multinationales et leurs rapports au territoire local étranger : une approche par les ressources", Management International, vol.16, $\mathrm{n}^{\circ} 1, \mathrm{p} .51-65$.

Ma X., Tong T. W., Fitza M. (2013), «How much does subnational region matter to foreign subsidiary performance? Evidence from Fortune Global 500 Corporations' investment in China», Journal of International Business Studies, vol. 44, nº 1, p. 66-87.

Mahnke V, Venzin M, Zahra S.A, (2007), "Governing Entrepreneurial Opportunity Recognition in MNEs : Aligning Interests and Cognition Under Uncertainty ", Journal of Management Studies, vol. 44, $\mathrm{n}^{\circ}$ 7, p. 1278-1298.

Marty F, (2005), «Politiques d'attractivité des territoires et règles européennes de concurrence - Le cas des aides versées par les aéroports aux compagnies aériennes ", Revue de l'OFCE, vol.94, p.97-125.

Maskell P, Malmberg A, (2007), «Myopia, knowledge development and cluster evolution", Journal of Economic Geography, vol. 7, n5 5 , p. 603-618.

Mataloni R. J. (2011), «The structure of location choice for new U.S. manufacturing investments in Asia-Pacific», Journal of World Business, vol. 46, n², p. 154-165.

MaYer T, Mucchielli J.L, (1999), «La localisation à l'étranger des entreprises multinationales : une approche d'économie géographique hiérarchisée appliquée aux entreprises japonaises en Europe», Économie et Statistique, n 326-327, p. 159-176.

Mayrhofer U, Urban S, (2011), Management international - Des pratiques en mutation, Pearson Education France, Paris.

McCann P, Mudambi R, (2005), "Analytical differences in the economics of geography : the case of the multinational firm ", Environment and Planning A, vol.37, n¹0, p. 1857-1876.

MÉTAIs E, (2004), Stratégie et ressources de l'entreprise, Economica, Paris.

Meyer K. E, Mudambi R, Narula R, (2011), «Multinational Enterprises and Local Contexts : The Opportunities and Challenges of Multiple Embeddedness", Journal of Management Studies, vol. $48, \mathrm{n}^{\circ} 2$, p. 235-252.

Meyer K. E., Nguyen H. V. (2005), «Foreign Investment Strategies and Sub-national Institutions in Emerging Markets : Evidence from Vietnam», Journal of Management Studies, vol. 42, nº1, p. 63-93.

Michalet C.A, (1999), La séduction des nations ou comment attirer les investissements, Economica, Paris.

Miles M.B, Huberman, A.M, (1991), Analyse des données qualitatives : recueil de nouvelles méthodes, De Boeck.

Mucchielli J.L, Puech F, (2003), «Internationalisation et localisation des firmes multinationales : l'exemple des entreprises françaises en Europe ", Economie et Statistique, n³63-364-365, p.129-144.
MucChielLi, J.L. (1998). Multinationales et mondialisation, Éditions du Seuil, Paris.

NARula R, Dunning J.H, (2010), «Multinational Enterprises, Development and Globalization : Some Clarifications and a Research Agenda», Oxford Development Studies, vol. 38, n³, p. 263-287.

Nell P.C, Аmbos B, Schlegelmilch B.B, (2011), «The MNC as an externally embedded organization : An investigation of embeddedness overlap in local subsidiary networks ", Journal of World Business, vol. 46, nº, p. 497-505.

Noisette P, Vallerugo F, (2010), Un monde de villes. Le marketing des territoires durables, Editions de l'Aube, Clermont-Ferrand.

Penrose E.T, (1963), Facteurs, conditions et mécanismes de la croissance de l'entreprise, Editions Hommes et Techniques, traduction de la version anglaise The Theory of the Growth of the Firm, Oxford, Basil Blackwell, 1959, par S, Mayret; L, Mayret.

Perrat J, Zimmermann J.B, (2003), «Stratégies des firmes et dynamiques territoriales", dans Dupuy, C., Burmeister, A. (dir.). Entreprises et territoires - Les nouveaux enjeux de la proximité, Les Etudes de la Documentation Française, Economie, p. 1-24.

Porter M.E, (1990), The Competitive Advantage of Nations, MacMillan London.

Porter M.E, (2000), «Location, Competition, and Economic Development : Local Clusters in a Global Economy», Economic Development Quarterly, vol.14, n¹, p.15-34.

Prahalad C.K, Hamel G, (1990), «The Core Competencies of the Corporation", Harvard Business Review, vol.68, n³, p.79-93.

Rugman A, Verbeke A, (2009), «Location, competitiveness, and the multinational enterprise», In Rugman, A. (Ed.). The Oxford Handbook of International Business - 2nd edition, Oxford : Oxford University Press, p. 146-182.

Rugman A, Verbeke A, Yuan W, (2011), «Re-conceptualizing Bartlett and Ghoshal's Classification of National Subsidiary Roles in the Multinational Enterprise ", Journal of Management Studies, vol. 48, n², p. 253-277.

Sachwald F, Perrin S, (2003), Multinationales et développement: le rôle des politiques nationales, Magellan \& Cie, France.

Sergot B, (2004), «Les déterminants des décisions de localisation - Les créations de nouveaux sites des entreprises françaises de l'industrie et des services ", Thèse de Doctorat en Sciences de Gestion, I.A.E, Université Paris I - Panthéon Sorbonne, 6 décembre, Paris.

Sergot B, (2007), «La personnalisation des décisions de localisation : Le cas des créations de nouveaux sites dans les secteurs de l'industrie manufacturière et de l'édition de logiciels", Finance Contrôle Stratégie, vol. 10, n4, p. 111-140.

Torre A, Beuret J.E, (2012), Proximités territoriales, Paris : Economica.

Veltz P, (1996), Mondialisation, villes et territoires : l'économie archipel, PUF, Paris.

Verbeke A, Chrisman J.J, Yuan W, (2007), «A Note on Strategic Renewal and Corporate Venturing in the Subsidiaries of Multinational Enterprises", Entrepreneurship Theory and Practice, vol. $31, \mathrm{n}^{\circ} 4$, p. 585-600.

Yin R.K, (2003), Case Study Research : Design and Methods, 3rd ed., Sage Publication, Newbury Park. 